\title{
Hypoxemia, high alveolar-arterial gradient and bubbles in both sides of heart: a case of Hepatopulmonary Syndrome in the setting of Covid-19 Pandemic.
}

\author{
Javier Torres ${ }^{1}$, Arturo Quevedo ${ }^{2}$, and Fernando Untiveros ${ }^{1}$ \\ ${ }^{1}$ National Hospital Edgardo Rebagliati Martins \\ ${ }^{2}$ National Hospital Alberto Sabogal Sologuren
}

November 4, 2020

\begin{abstract}
36-year-old man with past medical history of liver cirrhosis presented to the ED during COVID-19 pandemic peak with a history of shortness of breath on minimal exertion, mild cough and fever. Findings on physical examination included hypoxic, tachypnoea, ascites, pedal edema and digital clubbing. No abnormal sounds were heard on lung and heart auscultation. Due to the high suspicion of COVID-19, tests were performed. Thoracic CT scan demonstrated neither ground glass opacities nor consolidation. SARS-CoV-2 testing was negative. ABG showed a $\mathrm{PaO} 2$ of $51 \mathrm{~mm} \mathrm{Hg}$ with an A-a gradient of $68 \mathrm{~mm}$ Hg. A targeted history revealed platypnoea-orthodeoxia syndrome. Contrast echocardiography with saline was consistent with a diagnosis of hepatopulmonary syndrome. Patient was discharged with long-term oxygen. Currently, the patient is on the waiting list for a liver transplant.
\end{abstract}

\section{TITLE PAGE}

1. Title: Hypoxemia, high alveolar-arterial gradient and bubbles in both sides of heart: a case of Hepatopulmonary Syndrome in the setting of Covid-19 Pandemic.

2. Name of Authors: Torres-Valencia Javier M.D. ${ }^{1,2}$, Quevedo-Mori Arturo M.D. ${ }^{3}$, Untiveros-Mayorga Fernando M.D. ${ }^{1}$

3. Institutional addresses:

4. Department of Cardiology, Edgardo Rebagliati Martins National Hospital. 490 Rebagliati Avenue, Jesus Maria District, Lima, Peru. Postal code 15072.

5. Alberto Hurtado School of Medicine. Cayetano Heredia University. 430 Honorio Delgado, San Martín de Porres District, Lima, Peru. Postal Code 15102.

6. Emergency Department, Alberto Sabogal Sologuren National Hospital, 1081 Colina Jiron, Bellavista District, Callao, Peru, Postal code 07011.

7. Address for correspondence: Javier Torres Valencia, 1750 Almirante Guisse Street Apartment 603, Lince, Lima, Perú. Telephone: +51 943270905. E-mail: javier.tval@gmail.com. Fax: none.

8. Contributors: Conception or design of the case report: JTV. Data collection: JTV, AQM, FUM. Drafting the case report: JTV, AQM. Critical revision of the case report: FUM. Final approval of the version to be published: JTV, AQM, FUM.

\section{CASE SUMMARY}

36-year-old man with past medical history of liver cirrhosis presented to the ED during COVID-19 pandemic peak with a history of shortness of breath on minimal exertion, mild cough and fever. Findings on physical examination included hypoxic, tachypnoea, ascites, pedal edema and digital clubbing. No abnormal sounds 
were heard on lung and heart auscultation. Due to the high suspicion of COVID-19, tests were performed. Thoracic CT scan demonstrated neither ground glass opacities nor consolidation. SARS-CoV-2 testing was negative. ABG showed a $\mathrm{PaO}_{2}$ of $51 \mathrm{~mm} \mathrm{Hg}$ with an A-a gradient of $68 \mathrm{~mm} \mathrm{Hg}$. A targeted history revealed platypnoea-orthodeoxia syndrome. Contrast echocardiography with saline was consistent with a diagnosis of hepatopulmonary syndrome. Patient was discharged with long-term oxygen. Currently, the patient is on the waiting list for a liver transplant.

\section{KEYWORDS}

Hepatopulmonary Syndrome, Platypnoea and Orthodeoxia Syndrome, Hypoxemia, COVID-19, Digital clubbing, Case report.

\section{Introduction}

Current SARS-Cov-2 virus pandemic (COVID-19 pandemic) challenges physicians to find diagnosis and effective treatment for ill patients with hypoxemia. However, it is not the only cause, especially in patients with liver cirrhosis.

Hepatopulmonary Syndrome (HPS) has three components: liver disease, pulmonary vascular dilatation and a defect in oxygenation ${ }^{1}$. This syndrome presents insidiously and requires a high degree of clinical suspicion.

We report the clinical course of a patient, who initially presented hypoxemia in the pandemic peak, but after performing a focused history revealed severe HPS.

\section{Case presentation}

A 36-year-old Hispanic male with a past medical history of Child C cirrhosis presented in the pandemic peak with shortness of breath on minimal exertion, mild cough and fever. His history was significant for ligation of esophageal varices and prophylactic treatment with propranolol. In emergency department (ED), he was febrile to $38^{\circ} \mathrm{C}$ and hypoxic, requiring supplemental oxygen. He also reported upper right quadrant abdominal pain and jaundice. On physical examination we found tachypnoea, with normal breathing sounds and normal S1 and S2 heart sounds; no murmurs were appreciated. Abdominal distention, shifting dullness and fluid wave test were positive. Pedal edema, spider nevi and digital clubbing were present (figure 1). Normal mental status.

Due to COVID-19 pandemic had reached its peak and the threshold of suspicion was high, diagnostic COVID-19 tests were performed. Thoracic computed tomography (CT) scan demonstrated neither ground glass opacities nor consolidation (figure 2 and video 1 ). SARS-CoV-2 testing was negative.

Labs revealed lymphopenia at $0.16 \times 10^{3} / \mu \mathrm{L}$ (normal $0.9-5.2 \times 10^{3} / \mu \mathrm{L}$ ) and thrombocytopenia at $24 \mathrm{x}$ $10^{3} / \mu \mathrm{L}$ (normal $\left.130-400 \times 10^{3} / \mu \mathrm{L}\right)$. C-reactive protein at $7.8 \mathrm{mg} / \mathrm{dL}($ normal $<2.0 \mathrm{mg} / \mathrm{dL}$ ), ferritin at $224 \mathrm{ng} / \mathrm{mL}$ (normal 28-365 ng/mL). ABG, performed with the patient breathing ambient air while seated, showed $\mathrm{PaO}_{2} 51.2 \mathrm{mmHg}, \mathrm{PaCO}_{2} 23.6 \mathrm{mmHg}, \mathrm{FiO}_{2} 21 \%, \mathrm{PaO}_{2} / \mathrm{FiO}_{2} 243$, A-a gradient $68 \mathrm{mmHg}$ (patient temperature $37^{\circ} \mathrm{C}$ and 331 feet above sea level). Liver function test showed AST/ALT ratio 2.25, GGT 152 IU/L (normal 0-73 IU/L), total bilirubin $10.29 \mathrm{mg} / \mathrm{dL}$ (normal 0.3-1.2 mg/dL), conjugated bilirubin 7.16 $\mathrm{mg} / \mathrm{dL}$. Prothrombin time $19.7 \mathrm{sec}$ (normal 10.5-13 sec). Albumin $2 \mathrm{~g} / \mathrm{dL}$ (normal 3.2-4.8 g/dL).

During hospitalization, he continued on oxygen via nasal cannula for oxyhemoglobin saturation of $94 \%$. A focused history of his symptoms revealed increased shortness of breath on upright position and relieved by recumbency. Pulse oximetry in upright position was $87 \%$ while in supine position was $92 \%$. According to the previous findings, we suspected HPS, then a contrast-enhanced transthoracic echocardiography (TTE) with saline was performed, which showed no elevated right-sided heart pressures but abundant passage of microbubbles from the right chambers to the left chambers of the heart in the third cardiac cycle.

\section{(figure 3, 4, 5 and video 2).}

Home oxygen was provided to keep saturation between $92 \%$ and $95 \%$. Currently, the patient is on the waiting list for a liver transplant. 


\section{Discussion}

This case is an example of an infrequent cause of hypoxemia, in contrast to the typical deoxygenation in the COVID-19 pandemic scenario, with several clues of HPS.

HPS is a rare condition that can cause hypoxemia with specific findings. However, it is the most common cause of respiratory insufficiency in a patient with a chronic liver disease (occurs in $5 \%$ to $30 \%)^{2}$. This syndrome is characterized by abnormal oxygenation as a result of intrapulmonary vascular dilatation ${ }^{1}$.

Pathogenesis in HPS is complicated ${ }^{1,3,4}$. The hepatic injury/failure and portal hypertension increase releasing of endothelin-1 which in turn increases expression and activity of endothelial nitric oxide synthase eliciting higher production of nitric oxide. The latter develops intrapulmonary vascular dilatations. On the other hand, bacterial translocation, portosystemic shunt and hyperdynamic circulation produce systemic inflammation and recruitment of the macrophages in the lungs contributing to angiogenesis and vasodilation.

Alveolar-arterial gradient and the partial pressure of oxygen $(>15 \mathrm{mmHg}$ and $<80 \mathrm{mmHg}$, respectively) are reasonable criteria for suspected diagnosis while breathing room air in the sitting position at rest ${ }^{1,3}$. Also, the $\mathrm{PaO} 2$ suggest the severity of HPS. Mild HPS has a [?] $80 \mathrm{~mm} \mathrm{Hg}$ on air, moderate HPS has a PaO2 [?] 60 and $<80 \mathrm{~mm} \mathrm{Hg}$ on air, severe HPS has a $\mathrm{PaO} 2$ [?]50 and $<60 \mathrm{~mm} \mathrm{Hg}$ on air and very severe HPS has a $\mathrm{PaO} 2<50 \mathrm{~mm} \mathrm{Hg}$ on air or $<300 \mathrm{~mm} \mathrm{Hg}$ while the patient is breathing $100 \%$ oxygen. Our patient had a severe HPS ${ }^{1}$.

Platypnoea-orthodeoxia syndrome (POS) is another classic manifestation for HPS that is more specific, but not pathognomonic ${ }^{3}$. Platypnoea is an increase in the feeling of breathlessness when standing up which improves when lying down. Orthodeoxia is the decrease in $\mathrm{PaO} 2$ (typically by more than $4 \mathrm{~mm}$ of $\mathrm{Hg}$ ) or a reduction in saturations by more than $5 \% 1,3$. Our patient had a drop of $5 \%$. The syndrome is also described in patients with persistent foramen ovale ${ }^{5}$, the most common cause of POS reported ${ }^{6}$.

Contrast-enhanced TTE to characterize HPS is recommended ${ }^{4}$. Furthermore, this is less invasive and more available in the midst of the COVID-19 pandemic. After the administration of agitated saline in a peripheral vein in the arm, microbubble opacification of the left atrium within three to six cardiac cycles after rightatrial opacification indicates microbubble passage through an abnormally dilated vascular bed ${ }^{7}$. In our patient, these findings were consistent with a diagnosis of HPS.

Long-term supplemental oxygen was highly beneficial to our patient. However, the liver transplant is the only successful treatment ${ }^{4}$. At the time of writing he was on the waiting list for a liver transplant.

Acknowledgments: None.

Consent: The author/s confirm that written consent for submission and publication of this case report including image(s) and associated text has been obtained from the patient in line with COPE guidelines.

Conflict of interest: none declared.

\section{References:}

1. Rodriguez-Roisin R, Krowka MJ. Hepatopulmonary syndrome - A liver-induced lung vascular disorder. N Engl J Med. 2008;358(22):2378.

2. Krowka MJ, Fallon MB, Kawut S, Fuhrmann V, et al. International Liver Transplant Society Guidelines: Diagnosis and Management of Hepatopulmonary Syndrome and Portopulmonary Hypertension. Transplantation 2016;100:1440-1452.

3. Fuhrmann V, Krowka M. Hepatology Snapshot: Hepatopulmonary syndrome. J Hepatol. 2018;69:7445 .

4. Angeli P, Bernardi M, Villanueva C, et al. EASL Clinical Practice Guidelines for the management of patients with decompensated cirrhosis. J Hepatol. 2018;69(2):406-60. 
5. De Sousa Bispo J, Ramires I, Pestana J, et al. A case report of platypnea-orthodeoxia syndrome: an interplay of pressure and blood flow. Eur Hear J-Case Reports. 2019 Dec 1;3(4):1-4.

6. Agrawal A, Palkar A, Talwar A. The multiple dimensions of Platypnea-Orthodeoxia syndrome: A review. Respir Med. 2017 Aug;129:31-38.

7. Gupta SK, Shetkar SS, Ramakrishnan S, et al. Saline contrast echocardiography in the era of multimodality imaging - Importance of bubbling it right. Echocardiography. 2015;32(11):1707-19.

Figure legends:

Figure 1: Showing clubbed fingers.

Figure 2: Coronal CT image. Normal chest. CT: computed tomography.

Figure 3: TTE, capture of apical four chamber view at the first beat showing no bubbles in left side. TTE: transthoracic echocardiography.

Figure 4: TTE, capture of apical four chamber view at the third beat showing some bubbles in left side. TTE: transthoracic echocardiography.

Figure 5: TTE, capture of apical four chamber view at the fifth beat showing significant bubbles in left side. TTE: transthoracic echocardiography.

Video 1: Axial CT scan demonstrated neither ground glass opacities nor consolidation. CT: computed tomography.

Video 2: Contrast-enhanced TTE with saline, apical four chamber view showing the microbubble opacification of the left atrium within three to six cardiac cycles after right-atrial opacification.

TTE: transthoracic echocardiography.

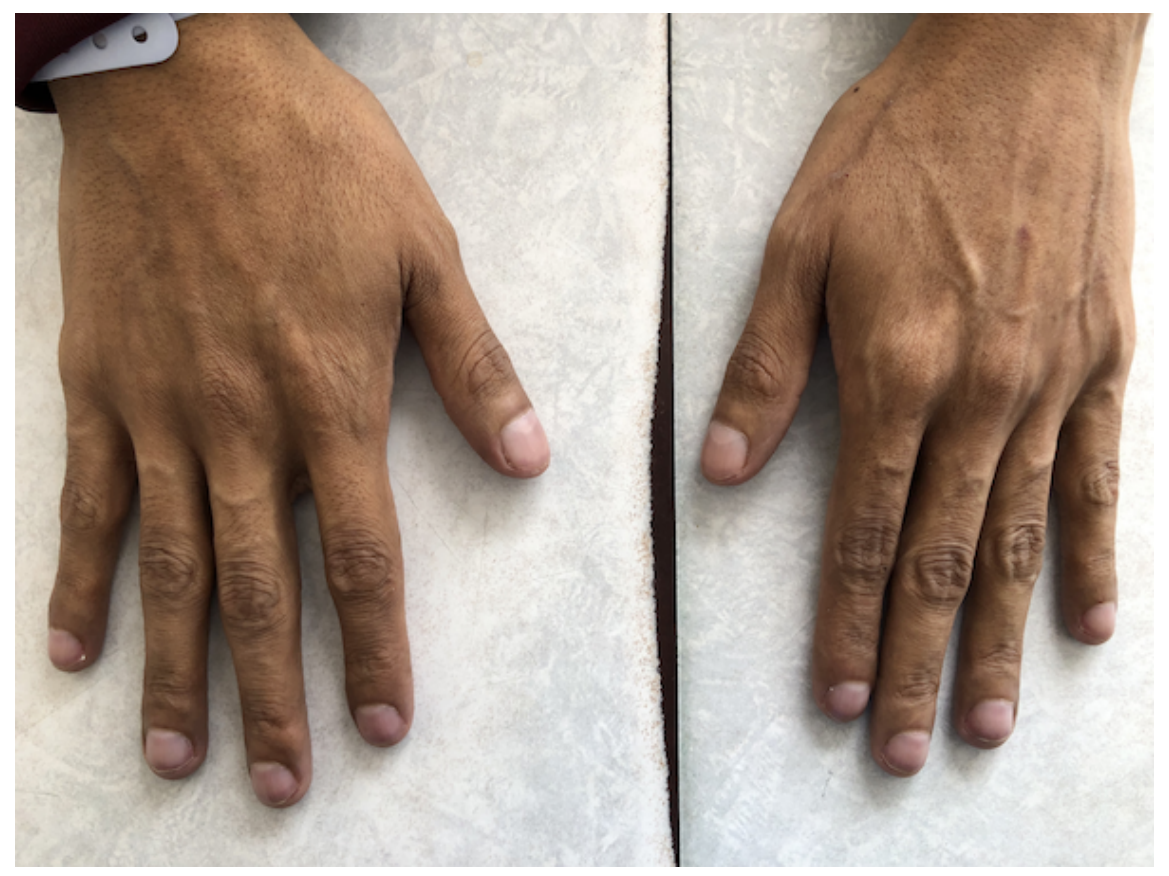



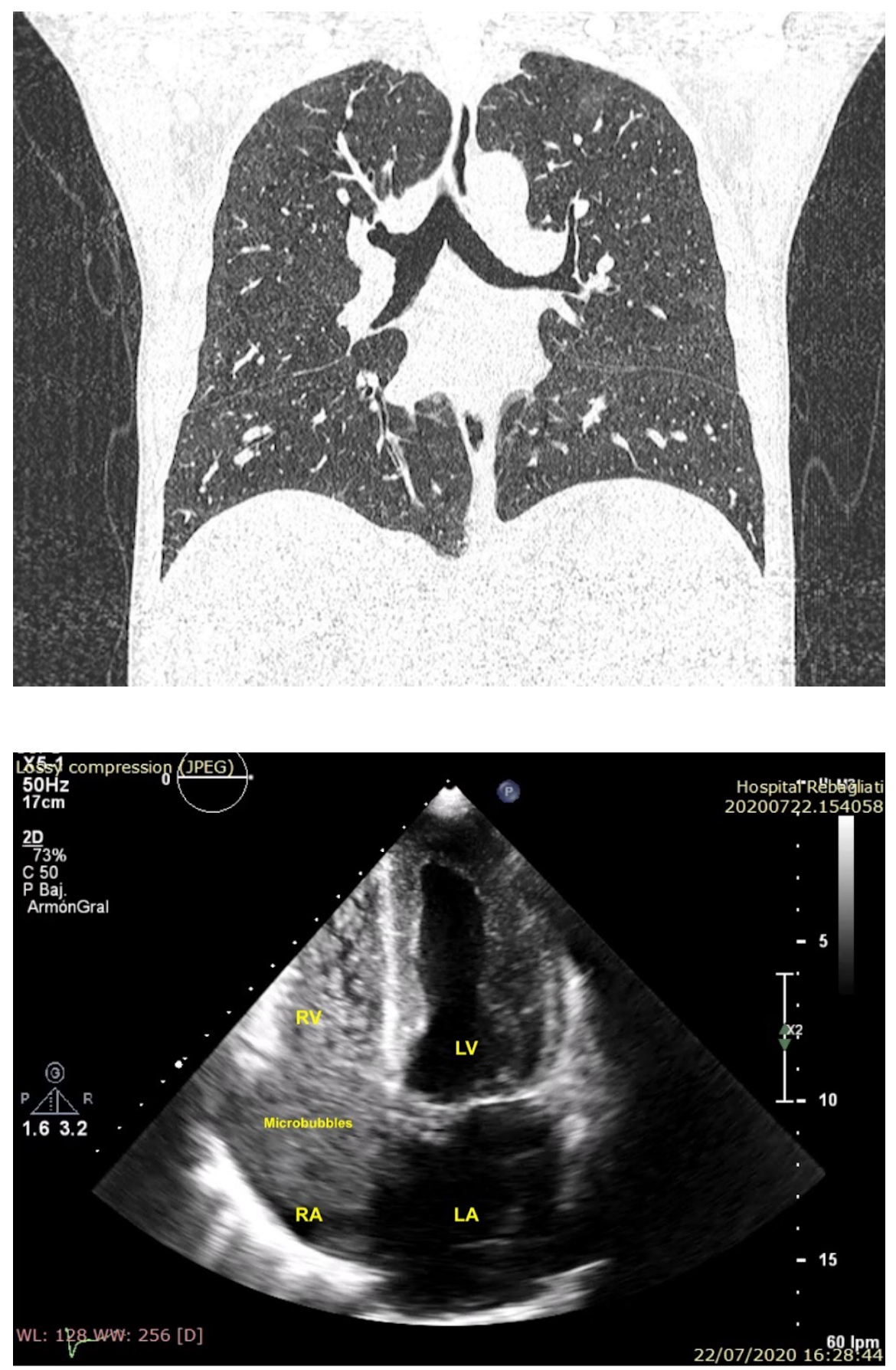

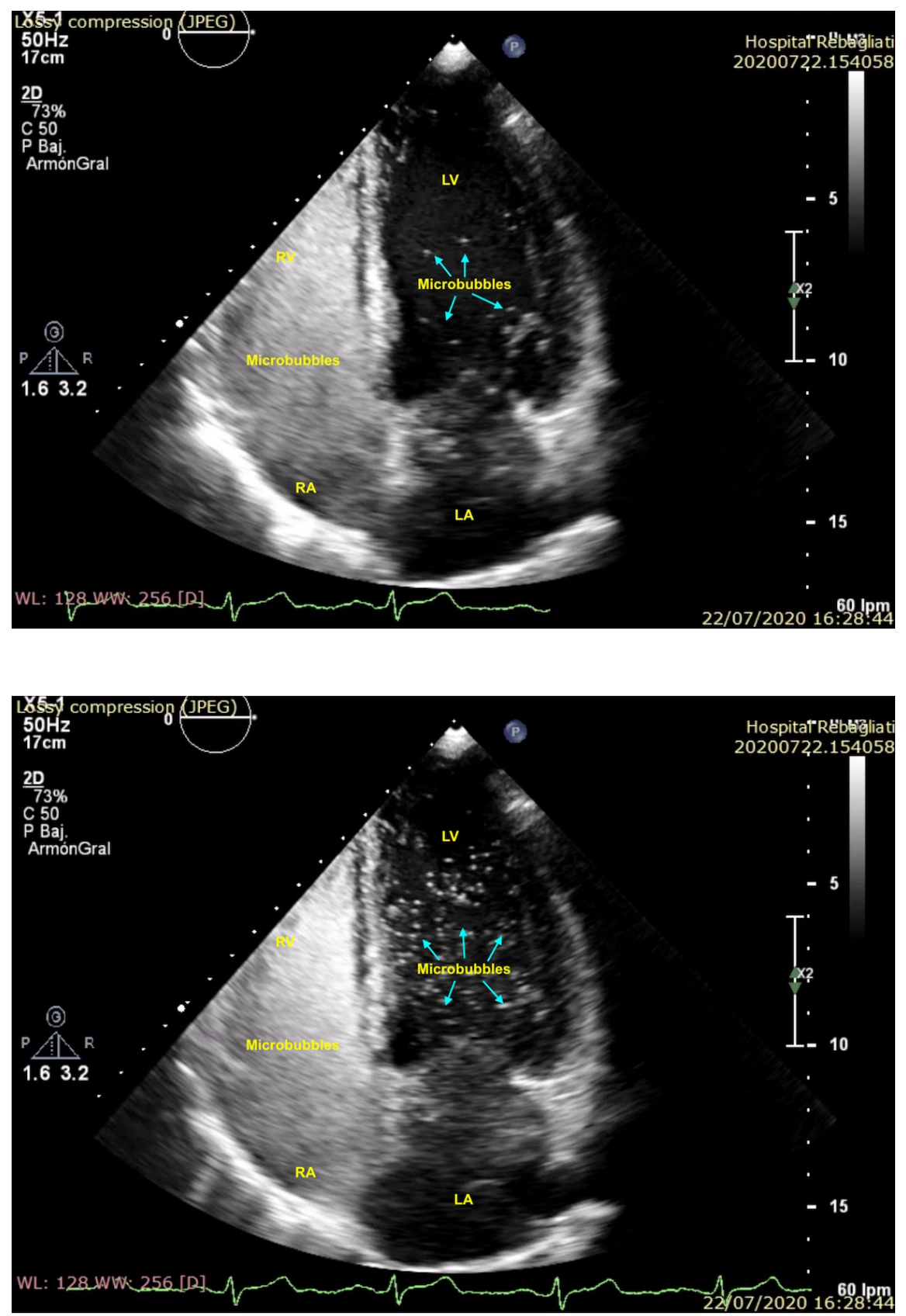